\title{
Dipeptidamide mit Süßstoffcharakter
}

\author{
B. Unterhalt und L. Kerckhoff
}

Institut für Pharmazeutische Chemie der Westfälischen Wilhelms-Universität Münster, Hittorfstr. 58-62, D-48149 Münster, Bundesrepublik Deutschland

\section{Dipeptidamides as Sweeteners}

Several L-Aspartyl-D-alanin-2-methylcycloalkylamides (9a-h) were synthesized and tested for their sweet quality.

(Keywords: Benzyl-N- $\alpha(Z)-L$-aspartyl-D-alanin-2-methylcycloalkylamides, hydrogenolysis to sweet tasting L-aspartyl-D-alanin-2-methylcycloalkylamides)

\section{Einleitung}

Nach Saccharin-Natrium und Natriumcyclamat kam L-Aspartyl-L-phenylalaninmethylester (1) unter dem Namen Aspartam in der Bundesrepublik Deutschland als dritter Süßstoff in den Handel. Aufgrund seiner Dipeptidstruktur ist Aspartam (1) weder hydrolysebeständig noch koch- und backstabil [1] und daher nur als Tafelsüßstoff einzusetzen. Molekülvariationen [2] führten u.a. zum L-Aspartyl-D-alanin-2,2,4,4-tetramethylthietanylamid Alitam (2), das seit 1986 in den USA gehandelt wird [3]. 2 ist etwa 2000 mal süßer als Saccharose, schmeckt wie Aspartam (1) rein süß und sollte in diätetischen Präparaten das phenylalaninhaltige $\mathbf{1}$ ersetzen können.<smiles>CC(=O)[C@H](Cc1ccccc1)NC(=O)[C@@H](N)CC(=O)O</smiles>

1<smiles>C[C@H](NC(=O)[C@@H](N)CC(=O)O)NC1C(C)(C)SC1(C)C</smiles>

2

Nach den bisherigen Ergebnissen zur Süßkraft 2- und 3-methylsubstituierter Cycloalkylsulfaminate [4] war es unser Ziel, monomethylsubstituierte Cycloalkylamine zur Herstellung alitamanaloger Verbindungen einzusetzen und die in Schema 1 skizzierte Strategie zu verfolgen. Die dort aufgefuhrten Methylcycloalkylamine (Tab.) wurden als Racemate sowie als Enantiomere verwendet, um Struktur-Wirkungs-Beziehungen zu studieren [5]. Die Synthese enantiomerenreiner 2-Methylcycloalkylamine gelang bei den cis-Formen durch hydrierende Aminierung der zugehörigen Ketone nach A.W. Frahm u. Mitarb. [6], bei den trans-Isomeren 
durch asymmetrische Hydroborierung-Aminierung von 2-Methylcycloalkenen mit Monoisopinocampheylboran als chiralem Reagenz nach H. C. Brown und Mitarb. [7]. Orientierende Tests an 3-Methylcycloalkylamiden zeigten, daß deren Süßungsgrade um den Faktor 20 niedriger liegen, so daß eine eingehende Untersuchung nicht lohnenswert schien [5].<smiles>[2H]NC(=O)[C@H](C)NC(=O)[C@H](N)CC(=O)O</smiles>

Schema 1

\section{Ergebnisse und Diskussion}

N- $\alpha$-Carbobenzoxy(Z-)-L-asparaginsäure-dibenzylester (3) wird selektiv mit 1 Äquivalent Natriumhydroxid in Wasser/1,4-Dioxan bei Raumtemperatur zu 4 hydrolysiert [8], 4 mit Chlorameisensäureisobutylester/ N-Methylmorpholin (NMM) [9] ins gemischte Anhydrid 5 übergeführt und durch Zugabe von D-Alanin-trimethylsilylester (6) ins Dipeptid Benzyl-N$\alpha(Z)$-L-aspartyl-D-alanin (7) umgewandelt.

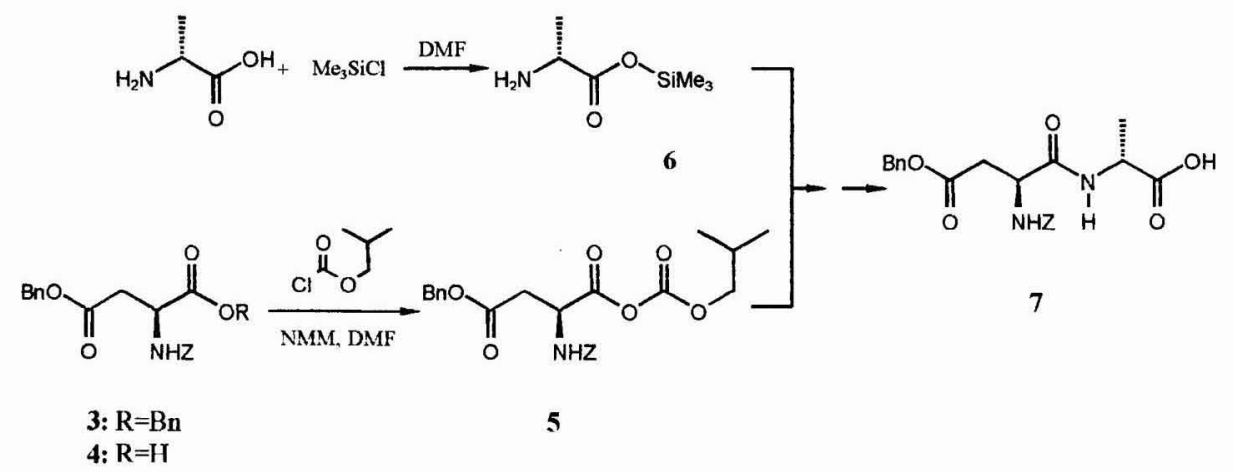

Die Reaktion von 7 mit Chlorameisensäureisobutylester/NMM bei $-15^{\circ} \mathrm{C}$ liefert nach Zugabe der 2-Methylcycloalkylamine a-h die geschützten Amide 8a-h, die durch katalytische Hydrogenolyse mit Pd/C in Methanol die gewünschten Produkte 9a-h ergeben (Tab.). Die Anhydridmethode ist nach eigener Erfahrung der sonst gebräuchlichen Verwendung von 1Ethyl-3-(3-dimethylaminopropyl)amino-carbodiimid (EDC) wegen weit geringerer Racemisierung überlegen [5].

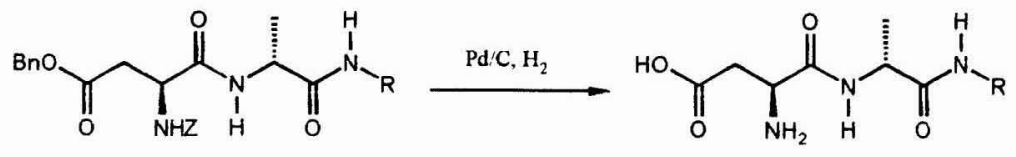




\begin{tabular}{|c|c|c|c|c|c|c|c|}
\hline Nr. & $-\mathrm{NH}-\mathrm{R}$ & SG & Fp $\left({ }^{\circ} \mathrm{C}\right)$ & Nr. & -NH-R & $S G$ & Fp $\left({ }^{\circ} \mathrm{C}\right)$ \\
\hline $9 a$ & & 250 & 178 & $9 e$ & & 75 & $198-200$ \\
\hline $9 \mathrm{~b}$ & & 150 & $145-153$ & 9f & & 125 & $218-220$ \\
\hline $9 c$ & & 200 & $205-207$ & $9 \mathrm{~g}$ & & 130 & $200-202$ \\
\hline 9d & & 250 & 210 & $9 \mathrm{~h}$ & & 300 & $199-200$ \\
\hline
\end{tabular}

Tab.: Süßungsgrade (SG) der synthetisierten L-Aspartyl-D-alanyl-2-methylcycloalkylamide 9

Vergleicht man die Süßungsgrade der cis-konfigurierten Verbindungen $\mathbf{9 a}, \mathbf{b}, \mathbf{e}, \mathbf{f}$ mit den gleichkonfigurierten Sulfaminaten- die trans-konfigurierten Sulfaminate wurden nicht untersucht-, so ist der höhere Süßungsgrad ( 20) bei den Analogen zu 9b und 9 f gegeben. Die beiden anderen sind entweder gar nicht oder höchstens schwach süß.

\section{Experimentelles}

Schmelzpunkte: Kofler-Heiztischmikroskop Reichert u. SMP-20 Büchi (unkorr.); ${ }^{1}$ H-NMR-u. ${ }^{13}$ C-NMR-Spektren: NMR-Spektrometer Gemini 200 Varian; Drehwerte: Polarimeter 341 Perkin Elmer; Elementaranalysen: CHN-Analyser 240 Perkin Elmer, CHNO-Rapid-Analyser Heraeus; Sensorik: Die Süßungsgrade werden durch Verkosten der wässer. Lösungen (Tafelwasser Cantrel ${ }^{\circledast}$, Raumtemp.) gegen Saccharose-Lösungen (3-5\%) ermittelt.

\section{Benzyl-N- $\alpha(Z)$-L-aspartyl-D-alanin(7) aus 5 und D-Alanin-trimethylsilylester (6)}

$6,3 \mathrm{~g}(71 \mathrm{mmol}) \mathrm{D}$-Alanin und 7,2 $\mathrm{g}(71 \mathrm{mmol}) \mathrm{Et}_{3} \mathrm{~N}$ werden in $100 \mathrm{ml} \mathrm{DMF}$ unter $\mathrm{N}_{2}$ gelöst, bei Raumtemp. mit 7,7 g (71 mmol) $\mathrm{ClSiMe}_{3}$ zu 6 umgesetzt und $1 \mathrm{~h}$ lang gerührt. In der Zwischenzeit werden $23,0 \mathrm{~g}(64 \mathrm{mmol}) 4$ (Schmp. $108^{\circ} \mathrm{C}$ [8]) und 7,2 $\mathrm{g}(71 \mathrm{mmol}) \mathrm{NMM}$ in einer Mischung aus $120 \mathrm{ml} \mathrm{DMF}$ und $120 \mathrm{ml}$ THF unter $\mathrm{N}_{2}$ gelöst und auf $-10^{\circ} \mathrm{C}$ abgekühlt. Man versetzt mit $9,6 \mathrm{~g}(70 \mathrm{mmol})$ Chlorameisensäureisobutylester, läßt $10 \mathrm{~min}$ lang bei $-10^{\circ} \mathrm{C}$ rühren und gibt zum Anhydrid $\mathbf{5}$ schnell die oben bereitete Lösung des D-Alanintrimethylsilylesters (6) hinzu. Nach $10 \mathrm{~min}$ bei $-10^{\circ} \mathrm{C}$ und $16 \mathrm{~h}$ bei Raumtemp. versetzt man 
die Reaktionsmischung mit $200 \mathrm{ml} \mathrm{0,2} \mathrm{M} \mathrm{HCl}$ und extrahiert dreimal mit je $100 \mathrm{ml} \mathrm{CHCl}$. Die vereinigten org. Phasen werden fünfmal mit je $70 \mathrm{ml} \mathrm{0,2} \mathrm{M} \mathrm{HCl}$ gewaschen, um DMF zu entfernen, über $\mathrm{Na}_{2} \mathrm{SO}_{4}$ getrocknet und i.Vak. eingeengt. DMF-Reste werden an der Ölpumpe entfernt, und der Rückstand wird nach Zusatz von $\mathrm{Et}_{2} \mathrm{O} 3 \mathrm{~h}$ lang gerührt. Die farblosen Kristalle des geschützten Dipeptids 7 werden abfiltriert und i. Vak. getrocknet. Ausbeute: $65 \%$, Schmp.: $148^{\circ} \mathrm{C}$. ${ }^{1} \mathrm{H}-\mathrm{NMR}\left(\mathrm{CDCl}_{3}, 200 \mathrm{MHz}\right): \delta(\mathrm{ppm})=1,34\left(\mathrm{~d}, \mathrm{~J}=7,2 \mathrm{~Hz},-\mathrm{CH}_{3}\right) ; 2,74$ (dd, $\mathrm{J}_{1}=16,2 \mathrm{~Hz}, \mathrm{~J}_{2}=7,9 \mathrm{~Hz}, 1 \mathrm{H}, \mathrm{CH}_{2}$ ) u. $2,93\left(\mathrm{dd}, \mathrm{J}_{1}=16,2 \mathrm{~Hz}, \mathrm{~J}_{2}=6,0 \mathrm{~Hz}, 1 \mathrm{H}, \mathrm{CH}_{2}\right) ; 4,35$ (q, J= 7,2 Hz, CH); 4,62 (dd, J $\left.=6,0 \mathrm{~Hz}, \mathrm{~J}_{2}=7,8 \mathrm{~Hz}, \mathrm{CH}\right) ; 5,08$ u. 5,09 (2s, 2x $\left.\mathrm{CH}_{2}\right) ; 7,31\left(\mathrm{~m}_{\mathrm{c}}\right.$, $\left.10 \mathrm{H}_{2 \mathrm{ra}}\right) ;{ }^{13} \mathrm{C}-\mathrm{NMR}\left(\mathrm{CDCl}_{3}, 50,3 \mathrm{MHz}\right): \delta(\mathrm{ppm})=17,76\left(\mathrm{CH}_{3}\right) ; 37,59\left(\mathrm{CH}_{2}\right) ; 49,56(\mathrm{CH}) ; 52,98$ $(\mathrm{CH}) ; 67,66$ u. $67,94\left(2 \times \mathrm{CH}_{2}\right) ; 128,76 ; 128,85 ; 129,02 ; 129,20 ; 129,47 ; 137,33 ; 138,02\left(\mathrm{C}_{\mathrm{ar}}\right)$; 156,34 (COOR); 171,$87 ; 172,58 ; 175,64(\mathrm{C}=\mathrm{O})$

Benzyl-N- $\alpha(Z)-L$-aspartyl-D-alanin-2-methylcycloalkylamide $8 \boldsymbol{a}$ - $\boldsymbol{h}$ :

$1,3 \mathrm{~g}$ (3 mmol) geschütztes Dipeptid 7 und 0,4 $\mathrm{g}(4 \mathrm{mmol})$ NMM werden in $20 \mathrm{ml} \mathrm{DMF}$ gelöst, auf $-10^{\circ} \mathrm{C}$ abgekühlt, mit $0,5 \mathrm{~g}(4 \mathrm{mmol})$ Chlorameisensäureisobutylester versetzt und 15 min lang gerührt. Man gibt $0,5 \mathrm{~g}(3,3 \mathrm{mmol})$ Methylcycloalkylamin- $\mathrm{HCl}$ (a-h) und $0,4 \mathrm{~g}(4$ $\mathrm{mmol}$ ) $\mathrm{Et}_{3} \mathrm{~N}$ in $20 \mathrm{ml} \mathrm{THF}$ hinzu, rührt $1 \mathrm{~h}$ lang bei $-10^{\circ} \mathrm{C}$ sowie $12 \mathrm{~h}$ lang bei Raumtemp., säuert die Reaktionsmischung mit $5 \times 20 \mathrm{ml} 0,2 \mathrm{M} \mathrm{HCl}$ an und extrahiert mit $\mathrm{CHCl}_{3}$. Die vereinigten org. Phasen werden mit $0,2 \mathrm{M} \mathrm{HCl}$ gewaschen, über $\mathrm{Na}_{2} \mathrm{SO}_{4}$ getrocknet, eingeengt und der ölige Rückstand in $\mathrm{Et}_{2} \mathrm{O}$ aufgenommen sowie so lange gerührt, bis die Kristallisation von 8a-h einsetzt. Das Dipeptid-Grundgerüst zeigt NMR-spektroskopisch folgende Signalbereiche: ${ }^{1} \mathrm{H}-\mathrm{NMR}\left(\mathrm{CDCl}_{3}, 200 \mathrm{MHz}\right): \delta(\mathrm{ppm})=1,34-1,37\left(\mathrm{~d}, \mathrm{~J}=7,0-7,2 \mathrm{~Hz}, \mathrm{CH}_{3}\right)$; 2,76-2,79 (dd, $\left.\mathrm{J}_{1}=16,8-17.4 \mathrm{~Hz}, \mathrm{~J}_{2}=5,4-5,8 \mathrm{~Hz}, 1 \mathrm{H}, \mathrm{CH}_{2}\right)$ u. 3,07-3,22 (dd, $\mathrm{J}_{1}=17,0-17,8 \mathrm{~Hz}$, $\left.\mathrm{J}_{2}=4,5-4,8 \mathrm{~Hz}, 1 \mathrm{H}, \mathrm{CH}_{2}\right) ; 4,38-4,44(\mathrm{q}, \mathrm{J}=7,0-7,6 \mathrm{~Hz}, \mathrm{CH}) ; 4,58-4,61\left(\mathrm{~m}_{\mathrm{c}}, \mathrm{CH}\right) ; 5,09-5,13$ (2s, $\left.2 \mathrm{CH}_{2}\right) ; 5,81-5,92,6,05-6,33$ u. 6,78-6,91 (bs oder d, $\left.3 \mathrm{NH}\right) ; 7,31-7,34\left(\mathrm{~m}, 10 \mathrm{H}_{\mathrm{ar}}\right)$. Die Peaks der Ringwasserstoffe finden sich bei den einzelnen Verbindungen. ${ }^{13} \mathrm{C}-\mathrm{NMR}\left(\mathrm{CDCl}_{3}\right.$, 50,3 MHz): $\delta(\mathrm{ppm})=36,2-36,6\left(\mathrm{CH}_{2}\right) ; 49,3-49,6\left(\mathbf{C H}-\mathrm{CH}_{3}\right) ; 51,4-51,6(\mathrm{CH}-\mathrm{N}) ; 67,0-67,7$ $\left(2 \mathrm{CH}_{2}\right) ; 128,2-128,8$ (i.a. $\left.5 \mathrm{C}_{\mathrm{ar}}\right) ; 135,3-136,0$ (i.a. $\left.2 \mathrm{C}_{\mathrm{ar}}\right) ; 170,1-171,8(4 \mathrm{C}=\mathrm{O})$. Das Methyl-CAtom des Alaninteils liegt bei 8a,b,e,f zwischen 17,6 und 18,0 ppm, bei 8c,d gegen 19,1 ppm und bei 8g,h gegen 18,2 ppm; die Angaben zu den Ringkohlenstoffen finden sich bei den einzelnen Verbindungen.

Benzyl-N- $\alpha(Z)-L$-aspartyl-D-alanin-(IR, 2S-methylcyclohexyl)amid (8a) Ausbeute: $41 \%$, Schmp.: $120-121^{\circ} \mathrm{C},[\alpha]^{20}{ }_{\mathrm{D}}:+32,85^{\circ}\left(\mathrm{c}=1,12\right.$; $\left.\mathrm{CHCl}_{3}\right) .{ }^{1} \mathrm{H}-\mathrm{NMR}\left(\mathrm{CDCl}_{3}, 200\right.$ 
MHz): $\delta(\mathrm{ppm})=0,80\left(\mathrm{~d}, \mathrm{~J}=7,0 \mathrm{~Hz}, \mathrm{CH}_{3}\right) ; 1,17-1,84(\mathrm{~m}, 9 \mathrm{H}) ; 4,00\left(\mathrm{~m}_{\mathrm{c}}, \mathrm{H}-1\right) .{ }^{13} \mathrm{C}-\mathrm{NMR}$ $\left(\mathrm{CDCl}_{3}, 50,3 \mathrm{MHz}\right): \delta(\mathrm{ppm})=16,41\left(\mathrm{CH}_{3}\right) ; 22,22(\mathrm{C}-5) ; 23,70(\mathrm{C}-4) ; 29,28(\mathrm{C}-3) ; 30,15(\mathrm{C}-$ 6); 33,64 (C-2); 49,80 (C-1).

\section{Benzyl-N- $\alpha(Z)$-L-aspartyl-D-alanin-(IS,2R-methylcyclohexyl)amid (8b)}

Ausbeute: $83 \%$, Schmp.: $149-151^{\circ} \mathrm{C},[\alpha]^{20}{ }_{\mathrm{D}}:+22,09^{\circ}\left(\mathrm{c}=1,24 ; \mathrm{CHCl}_{3}\right){ }^{1} \mathrm{H}-\mathrm{NMR}\left(\mathrm{CDCl}_{3}, 200\right.$ MHz): $\delta(\mathrm{ppm})=0,83\left(\mathrm{~d}, \mathrm{~J}=7,0 \mathrm{~Hz}, \mathrm{CH}_{3}\right) ; 1,18-1,85(\mathrm{~m}, 9 \mathrm{H}) ; 4,01\left(\mathrm{~m}_{\mathrm{c}}, \mathrm{H}-1\right)$. Bei

Verwendung von EDC als Kupplungsreagenz tritt teilweise Racemisierung im Alanin-Teil ein im ${ }^{1} \mathrm{H}$-NMR-Spektrum liegt die Methylgruppe jeweils als Dublett bei $\delta=1,35$ u. 1,44 ppm -

${ }^{13} \mathrm{C}-\mathrm{NMR}\left(\mathrm{CDCl}_{3}, 50,3 \mathrm{MHz}\right): \delta(\mathrm{ppm})=16,36\left(\mathrm{CH}_{3}\right) ; 22,24(\mathrm{C}-5) ; 23,66(\mathrm{C}-4) ; 29,59(\mathrm{C}-3)$; 30,15 (C-6); 33,64 (C-2); 49,82 (C-1).

Benzyl-N- $\alpha(Z)-L$-aspartyl-D-alanin-(IR,2R-methylcyclohexyl)amid (8c)

Ausbeute: $64 \%$, Schmp.: $172-174^{\circ} \mathrm{C},[\alpha]^{20}{ }_{\mathrm{D}}:+39,3^{\circ}\left(\mathrm{c}=1,23 ; \mathrm{CHCl}_{3}\right) .{ }^{1} \mathrm{H}-\mathrm{NMR}\left(\mathrm{CDCl}_{3}, 200\right.$ MHz): $\delta(\mathrm{ppm}) \doteq 0,87\left(\mathrm{~d}, \mathrm{~J}=6,4 \mathrm{~Hz}, \mathrm{CH}_{3}\right) ; 0,95-1,34(\mathrm{~m}, 4 \mathrm{H}) ; 1,58-1,93(\mathrm{~m}, 5 \mathrm{H}) ; 3,41\left(\mathrm{~m}_{\mathrm{c}}, \mathrm{H}-\right.$ 1). ${ }^{13} \mathrm{C}$ - NMR $\left(\mathrm{CDCl}_{3}, 50,3 \mathrm{MHz}\right): \delta(\mathrm{ppm})=17,86\left(\mathrm{CH}_{3}\right) ; 25,50(\mathrm{C}-5) ; 25,92(\mathrm{C}-4) ; 33,45(\mathrm{C}-$ 3); 34,43 (C-6); 38,23 (C-2); 54,41 (C-1).

Benzyl-N- $\alpha(Z)$-L-aspartyl-D-alanin-(IS, 2S-methylcyclohexyl)amid (8d)

Ausbeute: $70 \%$, Schmp.: $182-183^{\circ} \mathrm{C},[\alpha]^{20}{ }_{D}:+14,7^{\circ}\left(\mathrm{c}=1,24 ; \mathrm{CHCl}_{3}\right) .{ }^{1} \mathrm{H}-\mathrm{NMR}\left(\mathrm{CDCl}_{3}, 200\right.$ $\mathrm{MHz}): \delta(\mathrm{ppm})=0,86\left(\mathrm{~d}, \mathrm{~J}=6,0 \mathrm{~Hz}, \mathrm{CH}_{3}\right) ; 0,92-1,32(\mathrm{~m}, 4 \mathrm{H}) ; 1,62-1,94(\mathrm{~m}, 5 \mathrm{H}) ; 3,44\left(\mathrm{~m}_{\mathrm{c}}, \mathrm{H}-\right.$ 1). ${ }^{13} \mathrm{C}-\mathrm{NMR}\left(\mathrm{CDCl}_{3}, 50,3 \mathrm{MHz}\right): \delta(\mathrm{ppm})=17,99\left(\mathrm{CH}_{3}\right) ; 25,57(\mathrm{C}-5) ; 25,93(\mathrm{C}-4) ; 33,60(\mathrm{C}-$ 3); 34,40 (C-6); 38,48 (C-2); 54,32 (C-1).

\section{Benzyl-N- $\alpha(Z)-L$-aspartyl-D-alanin-(IR,2S-methylcyclopentyl)amid (8e)}

Ausbeute: $69 \%$, Schmp.: $141-142^{\circ} \mathrm{C},[\alpha]^{20}{ }_{\mathrm{D}}:+36,1^{\circ}\left(\mathrm{c}=1,26 ; \mathrm{CHCl}_{3}\right) .{ }^{1} \mathrm{H}-\mathrm{NMR}\left(\mathrm{CDCl}_{3}, 200\right.$ $\mathrm{MHz}): \delta(\mathrm{ppm})=0,83\left(\mathrm{~d}, \mathrm{~J}=7,2 \mathrm{~Hz}, \mathrm{CH}_{3}\right) ; 1,19-2,01(\mathrm{~m}, 7 \mathrm{H}) ; 4,20(\mathrm{q}, \mathrm{J}=6,8 \mathrm{~Hz}, \mathrm{H}-1) .{ }^{13} \mathrm{C}-$ $\mathrm{NMR}\left(\mathrm{CDCl}_{3}, 50,3 \mathrm{MHz}\right): \delta(\mathrm{ppm})=14,85\left(\mathrm{CH}_{3}\right) ; 21,90(\mathrm{C}-4) ; 31,41(\mathrm{C}-3) ; 32,05(\mathrm{C}-5)$; $36,19(\mathrm{C}-2) ; 53,66(\mathrm{C}-1)$.

Benzyl-N- $\alpha(Z)-L$-aspartyl-D-alanin-(1S,2R-methylcyclopentyl)amid (8f) Ausbeute: $68 \%$, Schmp : $152-153^{\circ} \mathrm{C},[\alpha]^{20}{ }_{\mathrm{D}}:+21,1^{\circ}\left(\mathrm{c}=1,33 ; \mathrm{CHCl}_{3}\right){ }^{1} \mathrm{H}-\mathrm{NMR}\left(\mathrm{CDCl}_{3}, 200\right.$ $\mathrm{MHz}): \delta(\mathrm{ppm})=0,82\left(\mathrm{~d}, \mathrm{~J}=7,0 \mathrm{~Hz}, \mathrm{CH}_{3}\right) ; 1,20-1,92(\mathrm{~m}, 7 \mathrm{H}) ; 4,20(\mathrm{q}, \mathrm{J}=7,4 \mathrm{~Hz}, \mathrm{H}-1) .{ }^{13} \mathrm{C}-$ 
NMR ( $\left.\mathrm{CDCl}_{3}, 50,3 \mathrm{MHz}\right): \delta(\mathrm{ppm})=14,54\left(\mathrm{CH}_{3}\right) ; 21,89(\mathrm{C}-4) ; 31,38(\mathrm{C}-3) ; 32,02(\mathrm{C}-5)$; 36,59 (C-2); 53,63 (C-1).

Benzyl-N- $\alpha(Z)$-L-aspartyl-D-alanin-(IR, 2R-methylcyclopentyl)amid (8g)

Ausbeute: $75 \%$, Schmp: $156^{\circ} \mathrm{C},[\alpha]^{20}{ }_{\mathrm{D}}:+17,1^{\circ}\left(\mathrm{c}=1,19 ; \mathrm{CHCl}_{3}\right) .{ }^{1} \mathrm{H}-\mathrm{NMR}\left(\mathrm{CDCl}_{3}, 200\right.$ $\mathrm{MHz}): \delta(\mathrm{ppm})=0,97\left(\mathrm{~d}, \mathrm{~J}=6,6 \mathrm{~Hz}, \mathrm{CH}_{3}\right) ; 1,11-2,12(\mathrm{~m}, 7 \mathrm{H}) ; 3,73(\mathrm{q}, \mathrm{J}=8,0 \mathrm{~Hz}, \mathrm{H}-1) .{ }^{13} \mathrm{C}-$ NMR $\left(\mathrm{CDCl}_{3}, 50,3 \mathrm{MHz}\right): \delta(\mathrm{ppm})=17,90\left(\mathrm{CH}_{3}\right) ; 22,09(\mathrm{C}-4) ; 32,37(\mathrm{C}-3) ; 32,43(\mathrm{C}-5)$; 41,28 (C-2); 57,76 (C-1).

Benzyl-N- $\alpha(Z)$-L-aspartyl-D-alamin-(1S,2S-methylcyclopentyl)amid (8h)

Ausbeute: $70 \%$, Schmp.: $152^{\circ} \mathrm{C},[\alpha]^{20}{ }_{\mathrm{D}}:+31,1^{\circ}\left(\mathrm{c}=0,6 ; \mathrm{CHCl}_{3}\right) .{ }^{1} \mathrm{H}-\mathrm{NMR}\left(\mathrm{CDCl}_{3}, 200 \mathrm{MHz}\right)$ : $\delta(\mathrm{ppm})=0,99\left(\mathrm{~d}, \mathrm{~J}=7,2 \mathrm{~Hz}, \mathrm{CH}_{3}\right) ; 1,11-2,25(\mathrm{~m}, 7 \mathrm{H}) ; 3,71(\mathrm{q}, \mathrm{J}=8,0 \mathrm{~Hz}, \mathrm{H}-1) \cdot{ }^{13} \mathrm{C}-\mathrm{NMR}$ $\left(\mathrm{CDCl}_{3}, 50,3 \mathrm{MHz}\right): \delta(\mathrm{ppm})=17,80\left(\mathrm{CH}_{3}\right) ; 22,15(\mathrm{C}-4) ; 32,37$ (C-3); 32,50 (C-5); 41,13 (C2); $57,86(\mathrm{C}-1)$.

Hydrogenolyse von 8a-h zu den L-Aspartyl-D-alanin-2-methylcycloalkylamiden 9a-h: $0,5 \mathrm{~g}$ geschütztes Peptidamid (8) werden in $100 \mathrm{ml}$ abs. $\mathrm{MeOH}$ gelöst und mit 0,3 g 10proz. $\mathrm{Pd} / \mathrm{C}$ bei Raumtemp. 16h lang in einer Parr-Apparatur hydriert. Man filtriert den Katalysator $\mathrm{ab}$, engt die Lösung i.Vak. ein und rührt den Rückstand über $\mathrm{Nacht}_{\text {in }} \mathrm{Et}_{2} \mathrm{O}$, wobei Kristallisation eintritt. Die Reinigung der mit kolloidalen Katalysatorresten behafteten Produkte erfolgt durch Ultrafiltration der wässer. Lösungen und anschließende Lyophilisation. Die Ausbeuten sind quantitativ, bei 9e etwa $90 \%$.

\section{L-Aspartyl-D-alanin-(1R, 2S-methylcyclohexyl)amid (9a)}

$[\alpha]^{20}{ }_{D}:+63,0^{\circ}\left(\mathrm{c}=0,4 ; \mathrm{H}_{2} \mathrm{O}\right) \cdot \mathrm{C}_{14} \mathrm{H}_{25} \mathrm{~N}_{3} \mathrm{O}_{4} \times 2 \mathrm{H}_{2} \mathrm{O}(335,41) B e r .:$ C 50,13 H 8,72 N 12,53; Gef.: C 49,88 H 8,63 N 12,97. ${ }^{1} \mathrm{H}-\mathrm{NMR}\left(\mathrm{CDCl}_{3}, 200 \mathrm{MHz}\right): \delta(\mathrm{ppm})=0,87(\mathrm{~d}, \mathrm{~J}=7,0 \mathrm{~Hz}$, $\left.\mathrm{CH}_{3}\right) ; 1,21-1,85$ (m, 9H); 1,34 (d, J= 7,0 Hz, $\left.\mathrm{CH}_{3}\right) ; 2,49-2,75\left(\mathrm{~m}, \mathrm{CH}_{2}\right) ; 3,93\left(\mathrm{~m}_{\mathrm{c}}, \mathrm{H}-1\right) ; 4,05$ $\left(\mathrm{m}_{\mathrm{c}}, \mathrm{CH}-\mathrm{N}\right) ; 4,39(\mathrm{q}, \mathrm{J}=7,2 \mathrm{~Hz}, 1 \mathrm{H}) \cdot{ }^{13} \mathrm{C}-\mathrm{NMR}\left(\mathrm{CDCl}_{3}, 50,3 \mathrm{MHz}\right): \delta(\mathrm{ppm})=16,48\left(\mathrm{CH}_{3}\right)$; 23,33 (C-5); 24,50 (C-4); 30,34 (C-3); 30,98 (C-6); 34,78 ( $\left.\mathbf{C H}_{2}\right)$; 38, 12 (C-2); 50,62 (C-CH $)$; 51,45 (C-N); 52,41 (C-1). - Die $\mathrm{CH}_{3}$-Gruppe des Alaninteils liegt fur 9a,b,e,f,g,h zwischen 18,47 und 18,85 ppm, die $\mathrm{C}=\mathrm{O}-$ Gruppen erscheinen gegen $170-175$ ppm. 


\section{L-Aspartyl-D-alanin-(IS, 2R-methylcyclohexyl)amid (9b)}

$[\alpha]^{20}$ : $-2,2^{\circ}\left(\mathrm{c}=0,4 ; \mathrm{H}_{2} \mathrm{O}\right) \cdot \mathrm{C}_{14} \mathrm{H}_{25} \mathrm{~N}_{3} \mathrm{O}_{4} \times 2 \mathrm{H}_{2} \mathrm{O}(335,41)$ Ber.: $\mathrm{C} 50,13 \mathrm{H} 8,72 \mathrm{~N} \mathrm{12,53;}$ Gef:: C 50,95 H 8,53 N 12,68. ${ }^{1} \mathrm{H}-\mathrm{NMR}\left(\mathrm{CDCl}_{3}, 200 \mathrm{MHz}\right): \delta(\mathrm{ppm})=0,88(\mathrm{~d}, \mathrm{~J}=5,8 \mathrm{~Hz}$, $\left.\mathrm{CH}_{3}\right) ; 1,04-1,86(\mathrm{~m}, 12 \mathrm{H}) ; 2,53-2,74\left(\mathrm{~m}, \mathrm{CH}_{2}\right) ; 3,92\left(\mathrm{~m}_{\mathrm{c}}, \mathrm{H}-1\right) ; 4,37\left(\mathrm{~m}_{\mathrm{c}}, 1 \mathrm{H}\right) ; 4,88\left(\mathrm{~m}_{\mathrm{c}}, \mathrm{CH}-\right.$ N). ${ }^{13} \mathrm{C}$-NMR: Das Spektrum stimmt praktisch mit dem von 9a überein.

\section{L-Aspartyl-D-alanin-(IR,2R-methylcyclohexyl)amid (9c)}

$[\alpha]^{20}{ }_{D}:+72,3^{\circ}\left(\mathrm{c}=0,4 ; \mathrm{H}_{2} \mathrm{O}\right) \cdot \mathrm{C}_{14} \mathrm{H}_{25} \mathrm{~N}_{3} \mathrm{O}_{4} \times \mathrm{H}_{2} \mathrm{O}(317,39)$ Ber:: C 52,98 H 8,57 N 13,24;

Gef.: C 52,89 H 8,50 N 12,97. ${ }^{1} \mathrm{H}-\mathrm{NMR}\left(\mathrm{CDCl}_{3}, 200 \mathrm{MHz}\right): \delta(\mathrm{ppm})=0,89(\mathrm{~d}, \mathrm{~J}=6,4 \mathrm{~Hz}$, $\left.\mathrm{CH}_{3}\right) ; 0,97-1,39(\mathrm{~m}, 6 \mathrm{H}) ; 1,34\left(\mathrm{~d}, \mathrm{~J}=7,2 \mathrm{~Hz}, \mathrm{CH}_{3}\right) ; 1,54-1,87(\mathrm{~m}, 3 \mathrm{H}) ; 2,59\left(\mathrm{dd}, \mathrm{J}_{1}=17,2 \mathrm{~Hz}\right.$, $\left.\mathrm{J}_{2}=9,0 \mathrm{~Hz}, 1 \mathrm{H}, \mathrm{CH}_{2}\right) ; 2,72\left(\mathrm{dd}, \mathrm{J}_{1}=17,2 \mathrm{~Hz}, \mathrm{~J}_{2}=5,1 \mathrm{~Hz}, 1 \mathrm{H}, \mathrm{CH}_{2}\right) ; 3,64\left(\mathrm{~m}_{\mathrm{c}}, \mathrm{H}-1\right) ; 4,05$ (dd, $\left.\mathrm{J}_{1}=8,6 \mathrm{~Hz}, \mathrm{~J}_{2}=5,1 \mathrm{~Hz}, 1 \mathrm{H}\right) ; 4,25\left(\mathrm{~m}_{\mathrm{c}}, \mathrm{CH}-\mathrm{N}\right) \cdot{ }^{13} \mathrm{C}-\mathrm{NMR}\left(\mathrm{CDCl}_{3}, 50,3 \mathrm{MHz}\right): \delta(\mathrm{ppm})=21,35$ $\left(\mathbf{C H}_{3}\right) ; 22,54\left(\mathbf{C H}_{3}\right) ; 29,55(\mathrm{C}-5) ; 29,79(\mathrm{C}-4) ; 37,09(\mathrm{C}-3) ; 38,53(\mathrm{C}-6) ; 41,04\left(\mathbf{C H}_{2}\right) ; 41,83$

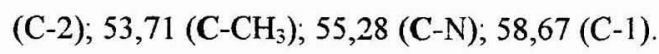

\section{L-Aspartyl-D-alanin-(1S, 2S-methylcyclohexyl)amid (9d)}

$[\alpha]^{20}{ }_{\mathrm{D}}:+32,0^{\circ}\left(\mathrm{c}=0,4 ; \mathrm{H}_{2} \mathrm{O}\right) . \mathrm{C}_{14} \mathrm{H}_{25} \mathrm{~N}_{3} \mathrm{O}_{4} \times 2,5 \mathrm{H}_{2} \mathrm{O}(344,42) \mathrm{Ber}$ : C 48,82 H 8,78 N 12,20; Gef:: C 48,83 H 8,25 N 11,90. ${ }^{1} \mathrm{H}-\mathrm{NMR}\left(\mathrm{CDCl}_{3}, 200 \mathrm{MHz}\right): \delta(\mathrm{ppm})=0,90(\mathrm{~d}, \mathrm{~J}=6,4 \mathrm{~Hz}$, $\left.\mathrm{CH}_{3}\right) ; 0,96-1,48(\mathrm{~m}, 5 \mathrm{H}) ; 1,38\left(\mathrm{~d}, \mathrm{~J}=7,2 \mathrm{~Hz}, \mathrm{CH}_{3}\right) ; 1,62-1,92(\mathrm{~m}, 4 \mathrm{H}) ; 2,53-2,71\left(\mathrm{~m}, \mathrm{CH}_{2}\right)$ 3,39 (m, H-1); 4,07 (mc, 1H); 4,32 (mc, CH-N). ${ }^{13} \mathrm{C}-\mathrm{NMR}\left(\mathrm{CDCl}_{3}, 50,3 \mathrm{MHz}\right): \delta(\mathrm{ppm})=$ $18,83\left(\mathrm{CH}_{3}\right) ; 25,07\left(\mathrm{CH}_{3}\right) ; 25,30(\mathrm{C}-5) ; 32,84(\mathrm{C}-4) ; 32,88$ (C-3); 37, 12 (C-6); 38,46 $\left(\mathrm{CH}_{2}\right)$; $39,76(\mathrm{C}-2) ; 48,17\left(\mathrm{C}-\mathrm{CH}_{3}\right) ; 48,31(\mathrm{C}-\mathrm{N}) ; 53,18(\mathrm{C}-1)$.

\section{L-Aspartyl-D-alamin-(1R,2S-methylcyclopentyl)amid(9e)}

$[\alpha]^{20}{ }_{\mathrm{D}}:+71,0^{\circ}\left(\mathrm{c}=0,5 ; \mathrm{H}_{2} \mathrm{O}\right) \cdot \mathrm{C}_{13} \mathrm{H}_{23} \mathrm{~N}_{3} \mathrm{O}_{4} \times 2,5 \mathrm{H}_{2} \mathrm{O}(330,39)$ Ber.: $\mathrm{C} 47,26 \mathrm{H} 8,54 \mathrm{~N} \mathrm{12,72}$, Gef.: C 46,99 H 8,68 N 12,50. ${ }^{1} \mathrm{H}-\mathrm{NMR}\left(\mathrm{CDCl}_{3}, 200 \mathrm{MHz}\right): \delta(\mathrm{ppm})=0,87(\mathrm{~d}, \mathrm{~J}=7,0 \mathrm{~Hz}$, $\left.\mathrm{CH}_{3}\right) ; 1,21-2,19(\mathrm{~m}, 7 \mathrm{H}) ; 1,36\left(\mathrm{~d}, \mathrm{~J}=7,0 \mathrm{~Hz}, \mathrm{CH}_{3}\right) ; 2,49-2,77\left(\mathrm{~m}, \mathrm{CH}_{2}\right) ; 4,10(\mathrm{~m}, 2 \mathrm{H}, \mathrm{H}-1 \mathrm{u}$. $\mathrm{CH}-\mathrm{N}) ; 4,36(\mathrm{q}, \mathrm{J}=7,0 \mathrm{~Hz}, \mathrm{CH}-\mathrm{N}){ }^{1.3} \mathrm{C}-\mathrm{NMR}\left(\mathrm{CDCl}_{3}, 50,3 \mathrm{MHz}\right): \delta(\mathrm{ppm})=14,88\left(\mathrm{CH}_{3}\right)$; 22,98 (C-4); 32,05 (C-3); 33, 10 (C-5); 38,29 ( $\left.\mathrm{CH}_{2}\right) ; 50,59\left(\mathrm{C}-\mathrm{CH}_{3}\right) ; 52,46(\mathrm{C}-\mathrm{N}) ; 54,46(\mathrm{C}-$ 1); C-2 nicht detektiert.

\section{L-Aspartyl-D-alanin-(1S,2R-methylcyclopentyl)amid(9f)}

$[\alpha]^{20}{ }_{D}:+16,1^{\circ}\left(\mathrm{c}=0,4 ; \mathrm{H}_{2} \mathrm{O}\right) \cdot \mathrm{C}_{13} \mathrm{H}_{23} \mathrm{~N}_{3} \mathrm{O}_{4} \times 2,5 \mathrm{H}_{2} \mathrm{O}(330,39)$ Ber.: C 47,26 H 8,54 N 12,72, 
Gef: C 47,14 H 8,60 N 12,50. 'H-NMR $\left(\mathrm{CDCl}_{3}, 200 \mathrm{MHz}\right): \delta(\mathrm{ppm})=0,87(\mathrm{~d}, \mathrm{~J}=5,4 \mathrm{~Hz}$, $\left.\mathrm{CH}_{3}\right) ; 1,01-2,15(\mathrm{~m}, 10 \mathrm{H}) ; 2,49-2,97\left(\mathrm{~m}, \mathrm{CH}_{2}\right) ; 3,92\left(\mathrm{~m}_{\mathrm{c}}, \mathrm{H}-1\right) ; 4,10\left(\mathrm{~m}_{\mathrm{c}}, \mathrm{CH}-\mathrm{N}\right) ; 4,36\left(\mathrm{~m}_{\mathrm{c}}\right.$, CH-N). ${ }^{13} \mathrm{C}-\mathrm{NMR}\left(\mathrm{CDCl}_{3}, 50,3 \mathrm{MHz}\right): \delta(\mathrm{ppm})=14,35\left(\mathrm{CH}_{3}\right) ; 21,43(\mathrm{C}-4) ; 30,43(\mathrm{C}-3)$; 31,46 (C-5); 36,11 (C-2); 38,40 ( $\left.\mathrm{CH}_{2}\right) ; 52,42(\mathrm{C}-\mathrm{N}) ; 52,52(\mathrm{C}-1)$; $\mathbf{C}-\mathrm{CH}_{3}$ nicht detektiert.

\section{L-Aspartyl-D-alanin-(1R,2R-methylcyclopentyl)amid(9g)}

$[\alpha]^{20}{ }_{D}:+32,3^{\circ}\left(\mathrm{c}=0,6 ; \mathrm{H}_{2} \mathrm{O}\right) \cdot \mathrm{C}_{13} \mathrm{H}_{23} \mathrm{~N}_{3} \mathrm{O}_{4} \times 2 \mathrm{H}_{2} \mathrm{O}(321,38)$ Ber.: $\mathrm{C} 48,58 \mathrm{H} 8,47 \mathrm{~N} 13,08$; Gef:: C 48,16 H 8,57 N 12,92. ${ }^{1} \mathrm{H}-\mathrm{NMR}\left(\mathrm{CDCl}_{3}, 200 \mathrm{MHz}\right): \delta(\mathrm{ppm})=0,89(\mathrm{~d}, \mathrm{~J}=6,5 \mathrm{~Hz}$, $\left.\mathrm{CH}_{3}\right) ; 1,09-1,94(\mathrm{~m}, 7 \mathrm{H}) ; 1,26\left(\mathrm{~d}, \mathrm{~J}=7,1 \mathrm{~Hz}, \mathrm{CH}_{3}\right) ; 2,50\left(\mathrm{dd}, \mathrm{J}_{1}=17,0 \mathrm{~Hz}, \mathrm{~J}_{2}=8,8 \mathrm{~Hz}, 1 \mathrm{H}\right.$, $\mathrm{CH}_{2}$ ); $2,63\left(\mathrm{dd}, \mathrm{J}_{1}=17,0 \mathrm{~Hz}, \mathrm{~J}_{2}=4,9 \mathrm{~Hz}, 1 \mathrm{H}, \mathrm{CH}_{2}\right) ; 3,54(\mathrm{q}, \mathrm{J}=8,0 \mathrm{~Hz}, \mathrm{H}-1) ; 3,97$ (q, J=4,7 $\mathrm{Hz}, \mathrm{CH}-\mathrm{N}) ; 4,21\left(\mathrm{~m}_{\mathrm{c}}, \mathrm{CH}-\mathrm{N}\right) \cdot{ }^{13} \mathrm{C}-\mathrm{NMR}\left(\mathrm{CDCl}_{3}, 50,3 \mathrm{MHz}\right): \delta(\mathrm{ppm})=18,37\left(\mathrm{CH}_{3}\right) ; 22,89$ (C-4); 32,82 (C-3); 33,39 (C-5); 38,06 (CH $)$; 41,94 (C-2); 50,71 (C-CH $)$; 52,39 (C-N); $59,01(\mathrm{C}-1)$.

\section{L-Aspartyl-D-alanin-(IS, 2S-methylcyclopentyl)amid(9h)}

$[\alpha]^{20}{ }_{D}:+77,5^{\circ}\left(\mathrm{c}=0,5 ; \mathrm{H}_{2} \mathrm{O}\right) \cdot \mathrm{C}_{13} \mathrm{H}_{23} \mathrm{~N}_{3} \mathrm{O}_{4} \times 2,5 \mathrm{H}_{2} \mathrm{O}(330,39)$ Ber.: C 47,26 H 8,54 N 12,72;

Gef:: C 47,08 H 8,67 N 12,65. ${ }^{1} \mathrm{H}-\mathrm{NMR}\left(\mathrm{CDCl}_{3}, 200 \mathrm{MHz}\right): \delta(\mathrm{ppm})=0.99(\mathrm{~d}, \mathrm{~J}=6,4 \mathrm{~Hz}$, $\left.\mathrm{CH}_{3}\right) ; 1,19-1,59(\mathrm{~m}, 2 \mathrm{H}) ; 1,33\left(\mathrm{~d}, \mathrm{~J}=7,0 \mathrm{~Hz}, \mathrm{CH}_{3}\right) ; 1,60-2,05$ (m, $\left.5 \mathrm{H}\right) ; 2,68-2,79\left(\mathrm{~m}, \mathrm{CH}_{2}\right)$; $3,63(\mathrm{q}, \mathrm{J}=8,0 \mathrm{~Hz}, \mathrm{H}-1) ; 4,09\left(\mathrm{~m}_{\mathrm{c}}, \mathrm{CH}-\mathrm{N}\right) ; 4,28(\mathrm{q}, \mathrm{J}=7,0 \mathrm{~Hz}, \mathrm{CH}-\mathrm{N}) \cdot{ }^{13} \mathrm{C}-\mathrm{NMR}\left(\mathrm{CDCl}_{3}, 50,3\right.$ $\mathrm{MHz}): \delta(\mathrm{ppm})=18,43\left(\mathrm{CH}_{3}\right) ; 22,89(\mathrm{C}-4) ; 32,88(\mathrm{C}-3) ; 33,46(\mathrm{C}-5) ; 38,00\left(\mathrm{CH}_{2}\right) ; 41,85(\mathrm{C}-$ 2); 50,77 (C- $\left.\mathrm{CH}_{3}\right) ; 52,30(\mathrm{C}-\mathrm{N}) ; 59,02(\mathrm{C}-1)$.

\section{Literatur}

1 Langguth, P., Alder, R., Merkle, H.P. (1991), Pharmazie 46, 188.

2 Brennan, T. M., Hendrick, M. E., US Pat. 4.411.925, s. (1984), C. A. 101, 22238; Eur. Pat. Appl. EP 34.876, s. (1982) C. A. 96, 104780.

3 Feinstein, R. D., Polinsky, A., Douglas, A. J., Beijer, C. M. G. F., Chadba, R. K., Benedetti, E., Goodman, M. (1991), J. Am. Chem. Soc. 113, 3467.

4 Unterhalt, B., Böschemeyer, L. (1971), Z. Lebensm. Unters. Forsch. 145, 93; Unterhalt, B., Möllers, M. (1991), Arch. Pharm. (Weinheim) 324, 525.

5 Kerckhoff, L. (1997), Diss., Univ. Münster.

6 Knupp, G., Frahm, A. W. (1984), Chem. Ber. 117, 2076; Wiehl, W., Frahm A. W. (1986), Chem. Ber. 119, 2668.

7 Brown, H. C., Kim, K.-W., Cole, T. E., Singaram, B. (1986), J. Am. Chem. Soc. 108, 6761; Fernandez, E., Hooper, M. W., Knight, F. I. (1997), Chem. Commun., 173.

8 Berger, A., Katchalski, E. (1951), J. Am. Chem. Soc. 73, 4087.

9 Anderson, G. W., Zimmerman, J. E., Callahan, F. M. (1967), J. Am. Chem. Soc. 89, 5012. 